\title{
ICA Based Face Recognition Robust to Partial Occlusions and Local Distortions
}

\author{
Jongsun Kim, Jongmoo Choi, and Juneho Yi \\ School of Information \& Communication Engineering \\ Sungkyunkwan University \\ 300, Chunchun-dong, Jangan-gu Suwon 440-746, Korea \\ \{jskim,jmchoi,jhyi\}@ece.skku.ac.kr
}

\begin{abstract}
We propose a novel method using a perfectly local facial representation based on ICA. We named our method "LS-ICA method". In the LS-ICA method, basis images are made from their corresponding ICA basis images simply by removing non-salient regions. The LS-ICA basis images display perfectly local characteristics because they contain only locally salient feature regions. This enables us to effectively realize the idea of "recognition by parts" for face recognition. Experimental results using AT\&T, Harvard, FERET and AR databases show that the recognition performance of the LS-ICA method outperforms that of PCA and ICA methods especially in the cases of facial images that have partial occlusions and local distortions such as changes in facial expression and at low dimensions.
\end{abstract}

\section{Introduction}

PCA and ICA are the most widely used subspace projection techniques in the face recognition research [2-3]. These techniques help to reveal low dimensional structures of patterns observed in high dimensional spaces. Figure 1 (a) and (b) show facial image representations using PCA and ICA basis images, respectively, that are computed from a set of images randomly selected from the AR database. PCA basis images display global properties in the sense that they assign significant weights to potentially all the pixels. This accords with the fact that PCA basis images are just scaled versions of global Fourier filters [13]. In contrast, ICA basis images are spatially more localized, highlighting salient feature regions corresponding to eyes, eyebrows, nose and lips.

The local property of ICA basis images makes the performance of ICA based recognition methods better than PCA based methods in terms of robustness to partial occlusions and local distortions, such as changes in facial expression, because spatially local features only influence small parts of facial images. Thus, ICA techniques have popularly been applied to the problem of face recognition [3-5]. However, ICA basis images do not display perfectly local characteristics in the sense that pixels that do not belong to locally salient feature regions still have some nonzero weight values. These pixel values in non-salient regions appear as noise and contribute to the degradation of the recognition performance as shown in the experimental results. 


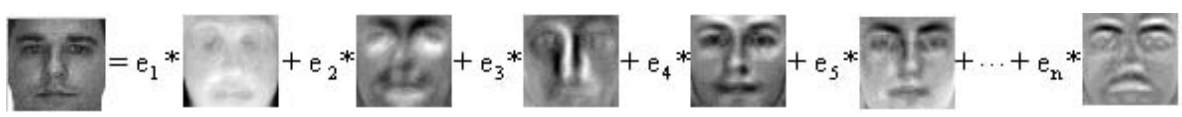

(a) PCA representation $=\left(\mathrm{e}_{1}, \mathrm{e}_{2}, \mathrm{e}_{3}, \mathrm{e}_{4}, \mathrm{e}_{5}, \ldots, \mathrm{e}_{\mathrm{n}}\right)$

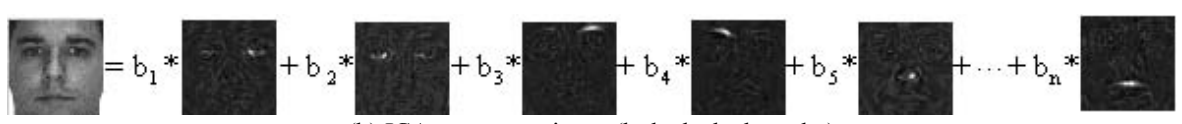

(b) ICA representation $=\left(b_{1}, b_{2}, b_{3}, b_{4}, b_{5}, \ldots, b_{n}\right)$

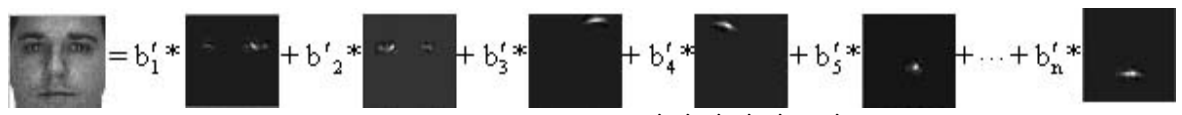

(c) LS-ICA representation $=\left(\mathrm{b}_{1}^{\prime}, \mathrm{b}_{2}^{\prime}, \mathrm{b}_{3}^{\prime}, \mathrm{b}_{4}^{\prime}, \mathrm{b}_{5}^{\prime}, \ldots, \mathrm{b}_{\mathrm{n}}^{\prime}\right)$

Fig. 1. Facial image representations using (a) PCA, (b) ICA and (c) LS-ICA basis images: A face is represented as a linear combination of basis images. The basis images were computed from a set of images randomly selected from the AR database. In the basis images of LS-ICA, non-salient regions of ICA basis images are removed. Using LS-ICA basis images, the concept of "recognition by parts" can be effectively implemented for face recognition

This research features a novel method using a perfectly local facial representation based on ICA. We named our method "LS-ICA (locally salient ICA) method". In the LS-ICA method, basis images contain only locally salient feature regions. The idea of "recognition by parts" can be effectively realized for face recognition using LS-ICA basis images since each LS-ICA basis image represents only locally salient regions. These regions correspond to important facial feature regions such as eyes, eyebrows, nose and lips. "Recognition by parts" [11-12] has been a popular paradigm in the object recognition research because the approach can be successfully applied to the problem of object recognition with occlusion. Our method for face recognition is characterized by two ideas. The first is the creation of LS-ICA basis images from ICA basis images and uses them to represent faces. Each LS-ICA basis image is made from its corresponding ICA basis image simply by removing non-salient regions. The second idea is to use ICA basis images in the decreasing order of class separability so as to maximize the recognition performance. We have tested three different distance metrics (L1 norm, L2 norm, and cosine angle) for PCA, ICA and LS-ICA method. The idea is simple but works great. Experimental results show that LS-ICA performs better than PCA and ICA, especially in the cases of partial occlusions and local distortions such as changes in facial expression. In addition, the performance improvement of LS-ICA over ICA based methods is much greater as we decrease the dimensionality (i. e., the number of basis images used).

The rest of this paper is organized as follows. Section 2 describes ICA versus PCA in terms of spatial locality of features. Section 3 explains the proposed LS-ICA method. Section 4 presents experimental results.

\section{ICA versus PCA}

PCA and ICA are the most widely used subspace projection techniques that project data from a high-dimensional space to a lower-dimensional space [2-3]. PCA addresses only second-order moments of the input. It is optimal for finding a reduced representation that minimizes the reconstruction error, but it is not optimal for 
classification. ICA is a generalization of PCA that decorrelates the high-order statistics in addition to the second-order moments. Much of information about characteristic local structure of facial images is contained in the higher-order statistics of the images. Thus ICA, where the high-order statistics are decorrelated, may provide a more powerful representational basis for face recognition than PCA, where only the second-order statistics are correlated. Fig. 2. illustrates PCA and ICA axes for the same 2D distribution. PCA finds an orthogonal set of axes pointing in the directions of maximum covariance in the data, while ICA attempts to place axes pointing in the directions of spatially localized and statistically independent basis vectors [10].

As previously described, global properties of faces may be more easily captured by PCA than ICA. As shown in Figure 1, ICA basis images are more spatially localized and never overlap unlike their PCA counterpart [1]. Since spatially localized features only influence small parts of facial images, ICA based recognition methods are less susceptible to occlusions and local distortions than are global feature based methods such as PCA. We can compute ICA basis images using various algorithms such as InfoMax [3], FastICA [4] and Maximum likelihood [5].
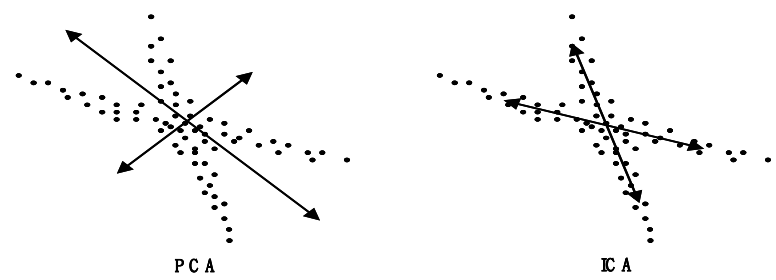

Fig. 2. PCA and ICA axes for an identical 2D data distribution [10]

\section{The LS-ICA (Locally Salient ICA) Method}

The LS-ICA method features the use of new basis images made from ICA basis images that are selected in the decreasing order of class separability. Only salient feature regions are contained in the LS-ICA basis images. As in most algorithms that employ subspace projection, the LS-ICA method computes a projection matrix, offline from a set of training images. Let $W_{l s-i c a}$ denote the projection matrix. The columns of $W_{l-i c a}$ are LS-ICA basis images. During recognition, given an input face image $\mathbf{x}$, it is projected to $\Omega^{\prime}=W_{l s-s i c}^{T} \mathbf{x}$ and classified by comparison with the vectors $\boldsymbol{\Omega}_{T}$ 's that were computed off-line from a set of training images.

First, we preprocess training images by applying histogram equalization and scale normalization, where the size of images is adjusted so that they have the same distance between two eyes. Second, we compute ICA basis images, using the FastICA algorithm [4]. The FastICA method computes the independent components that become uncorrelated by a whitening process and then maximizes non-Gaussianity of data distribution by using kurtosis maximization. We then compute a measure of class separability, $r$, for each ICA basis vector and sort the ICA basis vectors in the decreasing order of class separability [3]. To computer $r$ for each ICA basis vector, 
the between-class variability $\sigma_{\text {between }}$ and within-class variability $\sigma_{\text {within }}$ of its corresponding projection coefficients of training images are obtained as follows.

$$
\begin{gathered}
\sigma_{\text {between }}=\Sigma_{i}\left(M_{i}-M\right)^{2} \\
\sigma_{\text {within }}=\Sigma_{i} \Sigma_{j}\left(b_{i j}-M_{i}\right)^{2}
\end{gathered}
$$

$M$ and $M_{i}$ are the total mean and the mean of each class, and $b_{i j}$ is the coefficient of the $j^{\text {th }}$ training image in class $i$. The class separability, $r$, is then defined as the ratio

$$
r=\frac{\sigma_{\text {between }}}{\sigma_{\text {within }}}
$$

Third, we create LS-ICA basis images from the ICA basis images selected in the decreasing order of the class separability. This way, we can achieve both dimensional reduction and good recognition performance. To create an LS-ICA basis image, we apply a series of operations to its corresponding ICA basis image as shown in Fig. 4. In order to detect locally salient regions, we simply find extreme values by thresholding a histogram of pixel values (Figure $3(\mathrm{~b})$ ), followed by the application of morphological operations to find a blob region (Figure 3 (d)). As a result, we get an LS-ICA basis image (Figure 3 (e)) where only pixels in the blob regions have grey values copied from the corresponding pixels in the original ICA image. The values of the rest of the pixels in the image are set to zero. These LS-ICA basis images are used to represent facial images as shown in Figure 1 (c).

\section{Experimental Results}

We have used several facial image databases such as AT\&T [6], Harvard [7], FERET [8] and AR [9] databases in order to compare the recognition performance of LS-ICA with that of PCA and ICA methods. For fair comparisons with PCA and ICA based methods, PCA and ICA basis images were also used in the decreasing order of class separability, $r$. In the case of the ICA method, the recognition performance was greater when the basis images were ordered in terms of class separability. However, the PCA method did not show any noticeable performance difference between the ordering in the class separability and the orginal ordering in terms of eigenvalues.

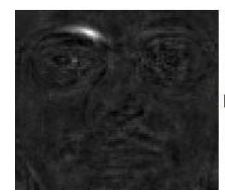

(a) ICA basis image

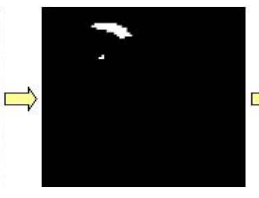

(b) detection of extreme values

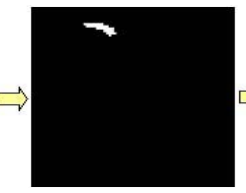

(c) erosion

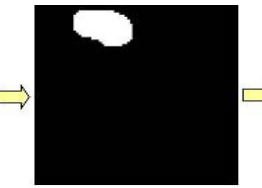

(d) dilation

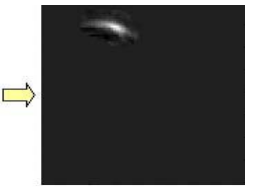

(e) LS-ICA basis image

Fig. 3. Illustration of creating an LS-ICA basis image 
Table 1. Facial databases used in the experiment

\begin{tabular}{|c|c|c|c|c|}
\hline Database & $\begin{array}{c}\text { The number of } \\
\text { total images }\end{array}$ & $\begin{array}{c}\text { The number of } \\
\text { persons }\end{array}$ & $\begin{array}{c}\text { The number of } \\
\text { training images }\end{array}$ & $\begin{array}{c}\text { The number of } \\
\text { testing images }\end{array}$ \\
\hline AT\&T & 400 & 40 & 200 & 200 \\
\hline Harvard & 165 & 5 & $82(83)$ & $83(82)$ \\
\hline FERET & 605 & 127 & 127 & 478 \\
\hline AR & 800 & 100 & 200 & 600 \\
\hline
\end{tabular}

Table 1 lists the number of training and testing images used in each facial image databases for the experiment. Fig. 4. shows example images from these databases. In the AT\&T database, all the images are taken against a dark homogeneous background and the subjects are in an up-right, frontal position with tolerance for some side movement. In Harvard database, a subject held his/her head steady while being illuminated by a dominant light source. In the FERET database, we have used a subset of the images of subjects under significantly different lighting and facial expression. The AR database contains local distortions and occlusions such as changes in facial expression and sunglasses worn.

All images were converted to 256 gray-level images and background regions were removed. We have also applied histogram equalization to both training and testing images in order to minimize variations of illumination. We have experimented using thirty different sets of training and testing images for each database. We have computed recognition performances for three different distance measures (L1, L2, cosine) since we are concerned with performance variations independent of the distance measure used [1].

Fig. 5. shows the recognition performances of PCA, ICA and LS-ICA methods for the four facial databases. The recognition rate of the LS-ICA method was consistently better than that of PCA and ICA methods regardless of distance measures used. ICA also consistently outperformed PCA except the case where the L1 measure was used for the FERET database. What is more interesting is that LS-ICA method performed better than the other methods especially at low dimensions. This property is very important when we need to store facial feature data in a low capacity storing devices such as smart cards and barcodes. To clearly show this, we displayed in Figure 6 the performance improvement of the LS-ICA method over the ICA method. The performance improvement was the greatest in the case of the AR database, as we expected. The AR database contains local distortions and occlusions such as sunglasses worn. The LS-ICA method that only makes use of locally salient information can achieve a higher recognition rate than ordinary ICA methods that are influenced by pixels not belonging to salient regions. The experimental results show that, especially at low dimensions, LS-ICA basis images better represent facial images than ICA basis images. 

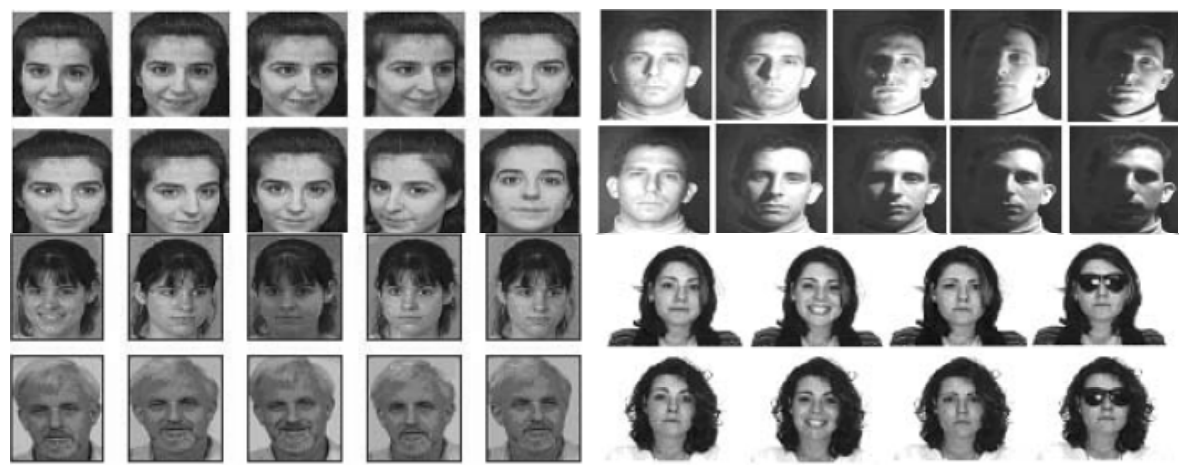

Fig. 4. Example images from AT\&T (top left), Harvard (top right), FERET (bottom left) and AR (bottom right) facial databases

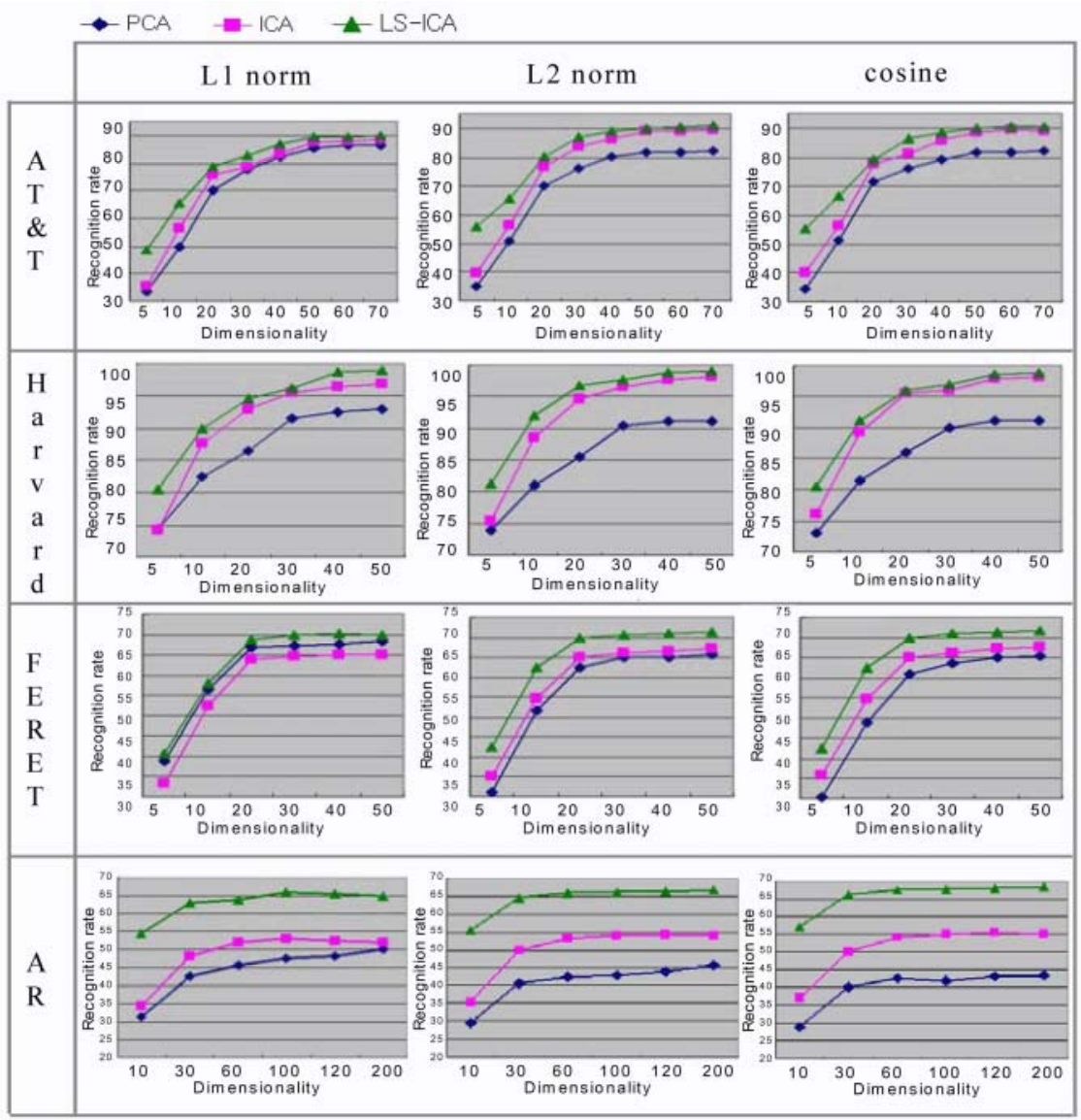

Fig. 5. The recognition performance of PCA, ICA and LS-ICA methods for the four facial databases. The recognition rates represent the average performance of the experiment using thirty different sets of training and testing images 


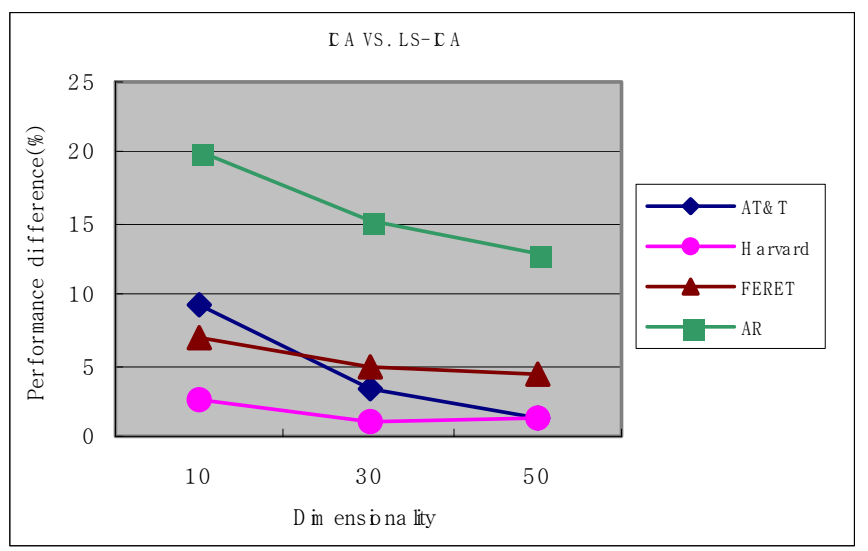

Fig. 6. The performance improvement of LS-ICA method over ICA method for the four facial databases. The performance improvement was the greatest in the case of AR database that contains local distortions and occlusions such as sunglasses worn

\section{Conclusions}

We have proposed the LS-ICA method that only employs locally salient information in order to maximize the benefit of applying the idea of "recognition by parts" to the problem of face recognition under partial occlusion and local distortion. The performance of the LS-ICA method was consistently better than the ICA method regardless of the distance measures used. As expected, the effect was the greatest in the cases of facial images that have partial occlusions and local distortions such as changes in facial expression.

\section{Acknowledgements}

This work was supported in part by grant No. R01-1999-000-00339-0 from the Basic Research Program KOSEF and BK21.

\section{References}

[1] K. Back, B. A. Draper, J. R. Beveridge, and K. She, "PCA vs ICA: A comparison on the FERET data set," Joint Conference on Information Sciences, Durham, N. C., 2002.

[2] M. A. Turk and A. P. Pentland, "Eigenfaces for recognition," Cognitive Neuroscience, vol.3, no.1, pp. 71-86, 1991.

[3] M. S. Bartlett, J. R. Movellan, and T. J. Sejnowski, "Face Recognition by Independent Component Analysis," IEEE Transaction on Neural Networks, Vol 13, pp. 1450-1464, 2002.

[4] Aapo Hyvarinen and Erki Oja, "Independent component analysis: a tutorial," http://www.cis.hut.fi/ aapo/papers/IJCNN99_tutorialweb/, 1999. 
[5] J. F. Cardoso, "Infomax and Maximum Likelihood for Source Separation," IEEE Letters on Signal Processing, vol. 4, pp. 112-114, 1997.

[6] http://www.uk.research.att.com/facedatabase.html.

[7] http://cvc.yale.edu/people/faculty/belhumeur.htm.

[8] P. J. Phillips, H. Moon, S. A. Rizvi, and P. J. Rauss, "The FERET Evaluation Methodology for Face Recognition Algorithms," Pattern Analysis and Machine Intelligence, vol. 22, pp. 1090-1104, 2000.

[9] M. Martinez and R. Benavente, "The AR face database," CVC Tech. Report \#24, 1998.

[10] M. S. Bartlett, "Face Image Analysis by Unsupervised Learning," Foreword by T. J. Sejnowski, Kluwer International Series on Engineering and Computer Science, Boston: Kluwer Academic Publishers, 2001.

[11] P. Pentland, "Recognition by parts," IEEE Proceedings of the First International Conference on Computer Vision, pp. 612-620, 1987.

[12] D. D. Lee and H. S. Seung, "Learning the parts of objects by non-negative matrix factorization," Nature, vol. 401, pp.788-791, 1999.

[13] J. Bell and T. J. Sejnowski, "The independent components of natural scenes are edge filters," Advance in Neural Information Processing Systems 9, 1997. 